\title{
Emergent geometry and path integral optimization for a Lifshitz action
}

\author{
A. Ahmadain and I. Klich \\ Department of Physics, University of Virginia, Charlottesville, Virginia 22903, USA
}

(Received 29 May 2020; accepted 2 April 2021; published 18 May 2021)

\begin{abstract}
Extending the background metric optimization procedure for Euclidean path integrals of twodimensional conformal field theories, introduced by Caputa et al. [Phys. Rev. Lett. 119, 071602 (2017), J. High Energy Phys. 11 (2017) 097], to a $z=2$ anisotropically scale-invariant (2+1)-dimensional Lifshitz field theory of a free massless scalar field, we find optimal geometries for static and dynamic correlation functions. For the static correlation functions, the optimal background metric is equivalent to an AdS metric on a Poincaré patch, while for dynamical correlation functions, we find Lifshitz like metric. This results suggest that a MERA-like tensor network, perhaps without unitarity, would still be considered an optimal background spacetime configuration for the numerical description of this system, even though the classical action we start with is not a conformal field theory.
\end{abstract}

DOI: 10.1103/PhysRevD.103.105013

\section{INTRODUCTION}

An important quest of many body physics is the search for efficient variational characterizations of correlated quantum systems. (for a review see, e.g., [1]). A class of tensor network states, particularly geared toward the description of scale-invariant systems, are called the multiscale entanglement renormalization ansatz (MERA) [2,3]. MERA is used to represent approximate ground states of 1D quantum spin chains at criticality described by 2D conformal field theory (CFT)[4]. The scale-invariance of the MERA network turned out to also play a special role in connecting it to holographic duals in the sense of the AdS/CFT correspondence [5]. Here, the bulk of a MERA network can be understood as a discrete realization of 3D anti-de Sitter space $\left(\mathrm{AdS}_{3}\right)$, identifying the extra holographic direction with the renormalization group $(\mathrm{RG})$ flow in the MERA [5].

The ground work for the connection between continuous tenor networks [6-8] and path integral optimization for $\mathrm{AdS}_{3} / \mathrm{CFT}_{2}$ was initially laid out in [9-11]. Recent work on the relationship between path integral optimization, different types of CFTs and complexity can be found in [12-15]. Motivated by the procedure of tensor network renormalization in [16], Caputa et al. [9,10], reinterpreted this connection as optimization of the background metric in the space of path integrals. Starting with flat Euclidean metric with a ultraviolet (UV) cutoff, they argued that their optimization procedure amounts to minimizing the Jacobian of the scale transformation

Published by the American Physical Society under the terms of the Creative Commons Attribution 4.0 International license. Further distribution of this work must maintain attribution to the author(s) and the published article's title, journal citation, and DOI. Funded by SCOAP ${ }^{3}$. for the path integral measure. In the conformally flat gauge, this translates to solving the equation of motion of the Liouville effective action from which they find that the $\mathrm{AdS}_{3}$ metric a Poincare patch $\mathrm{H}_{2}$ naturally emerges. This new approach is very appealing, as it suggests a concrete procedure connecting the AdS/CFT correspondence with numerical approaches to many body systems, such as the MERA tensor network [2,3,5,17].

In this paper, we extend the idea in $[9,10]$ to a nonrelativistic field theory, specifically to a $z=2$ anisotropically scale-invariant $(2+1)$-dimensional Lifshitz field theory of a free massless scalar field and show that the procedure can be successfully applied in systems of interest beyond a CFT. We show how natural geometries arise from the path integral optimization procedure. Our results are illustrated in Fig. 1.

\section{THE QUANTUM LIFSHITZ MODEL}

The quantum Lifshitz model is a canonical example of a $(2+1)$-dimensional Lifshitz field theory known [18].
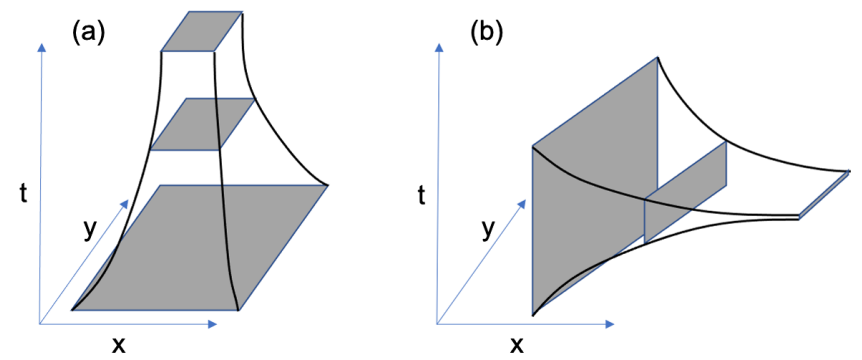

FIG. 1. The two geometries emerging for the quantum Lifhsitz model. (a) $\mathrm{An} \mathrm{AdS}_{3}$-like geometry arises when considering equal time correlation functions and (b) A Lifshitz metric that is optimal for computing correlation functions with a temporal component. 
This model describes a free massless scalar field with dynamical scaling exponent $z=2$ and represents an important example of a conformal quantum critical point. Different aspects of this theory have been studied and analyzed in [18-20]. For example, it emerges as the scaling limit of the square lattice quantum dimer model [20].

The quantum Lifshitz Hamiltonian [18] of a $z=2$ theory of a massless scalar field $\phi(t, \boldsymbol{x})$, where $\boldsymbol{x}=\{x, y\}$, in $2+1$ dimensions is given by

$$
H=\int d x\left\{\pi_{\phi}^{2}+\left(\Delta_{s} \phi\right)^{2}\right\} .
$$

The Euclidean action of the field $\phi(t, \boldsymbol{x})$ coupled to a background metric $g_{i j}$ is given by

$$
S=\int d \boldsymbol{x} d t N \sqrt{h}\left(N^{-2}\left(\partial_{t} \phi\right)^{2}+\left(\Delta_{s} \phi\right)^{2}\right),
$$

where $\Delta_{s}$ is the spatial Laplace-Beltrami operator

$$
\Delta_{s}=\frac{1}{\sqrt{h}} \partial_{i} h^{i j} \sqrt{h} \partial_{j},
$$

and $h_{i j}$ is the spatial component of the background metric [21].

$$
d s^{2}=N^{2} d t^{2}+h_{i j} d x^{i} d x^{j}
$$

where $N$ is called the lapse function. The action in (2) is invariant under the following foliation-preserving diffeomorphism transformations

$$
t \mapsto \tilde{t}(t), \quad \boldsymbol{x} \mapsto \tilde{\boldsymbol{x}}(\boldsymbol{x})
$$

and anistoropic Weyl scaling transformations

$$
N \rightarrow e^{z \sigma} N ; \quad h_{i j} \rightarrow e^{2 \sigma} h_{i j}, \quad i, j \neq t .
$$

As stated before, in [9], such a starting point led, via path integral optimization, to an AdS metric. The path integral optimization suggested in [9] looks for the extremal measure over all choices of the gauge $\sigma$, due to the Weyl anomaly in the model. Here we use the same structure, though with the appropriate anisotropic Weyl scaling exponent.

\section{GEOMETRY OPTIMIZATION FOR THE LIFSHITZ MODEL}

Here we ask the following question: what is the optimal geometry associated with a path integral computation of correlation functions in the quantum Lifshitz model? In contrast to the CFT case, due to the nonrelativistic nature of the model, equal time correlation functions and dynamical correlation functions should be treated differently. Indeed, we find two separate geometries associated with the optimal calculation, described in Fig. 1. For equal-time correlation functions, we consider Weyl transformations which are translationally invariant in space, but not in time, Fig. 1(a), covered by case (1) below.

Consider dynamical correlation functions on the other hand. To find the optimized geometry that describe two point functions, such as, say, $\left\langle\phi(t, \boldsymbol{x}) \phi\left(t, \boldsymbol{x}^{\prime}\right)\right\rangle$, we can choose the spatial axis $\boldsymbol{x}-\boldsymbol{x}^{\prime}$ to be our $y$ direction, due to spatial rotational invariance of the model. We concentrate therefore on the computation of the description of the state in the $t, y$ plane, and thus choose a Weyl scaling which is homogeneous in $t, y$, but can depend on the third coordinate $x$, Fig. 1(b) as explained in case (2) below.

Of particular interest to us in this paper, is the Weyl anomaly of this model which has first been computed holographically in [22] and by Baggio et al. in [23] using heat kernel expansion and the holographic renormalization methods in [24]. In [25,26], Lifshitz Weyl anomalies have been computed cohomologically in different dimensions and for different values of the dynamical scaling exponent $z$. In [27], the heat kernel expansion has been generalized to calculate effective actions and Weyl anomalies for Lifshitz field theories. A general framework for computing one loop effective action for Lifshitz theory via heat kernel coefficients has been presented in several places, see e.g., $[27,28]$.

We note that in contrast with [9], here, we do not start from the quantum effective action and then derive the equation of motion as they do but rather directly compute the variation in the Lifshitz effective action due to an infinitesimal transformation of the Weyl transformation parameter $\sigma$. Our starting point is a flat metric, deformed by a Weyl scaling, therefore $\sigma$ carries the entire information about the metric in the space of metrics we explore. We compute the variation of the effective action explicitly utilizing the particular structure of our metric and finally obtain differential equations for the scaling factor $\sigma$. Concretely, we compute the variation of the one loop effective action under $\sigma \rightarrow \sigma+\delta \sigma$. In this case (see, e.g., Appendix 5.A. in [29]),

$$
\delta W[\sigma]=\frac{1}{2} \int d \boldsymbol{r} \delta \sigma(\boldsymbol{r})\left\langle\boldsymbol{r}\left|e^{-\epsilon \rho D}\right| \boldsymbol{r}\right\rangle,
$$

where $\boldsymbol{r}=(\boldsymbol{x}, t), \rho(\boldsymbol{r})=\frac{1}{\sqrt{g(\boldsymbol{r})}}, \epsilon$ is the infinitesimal heat kernel "time" parameter, and $D=-\frac{1}{N \sqrt{h}} \partial_{t} N^{-1} \sqrt{h} \partial_{t}+$ $\frac{1}{N} \Delta_{s} N \Delta_{s}$ [23]. In our system, we fix our gauge so that $N=e^{2 \sigma}, h_{i j}=N \delta_{i j}$. In this case we have:

$$
D=\left(-\partial_{t}^{2}+\left(\partial_{x}^{2}+\partial_{y}^{2}\right)^{2}\right)
$$

We note that upon varying $\sigma$ we have $\delta D=-4 \delta \sigma D$. The $\epsilon \rightarrow 0$ behavior of (7) is dominated by the short distance behavior of the heat kernel $\left\langle\boldsymbol{r}\left|e^{-\epsilon \rho D}\right| \boldsymbol{r}\right\rangle$. 
Now, as promised, we specialize to cases where, $\sigma$ depends either on the time coordinate $t$ alone, or on one of the spacial coordinates, say $x$. Denoting $\rho=e^{-4 \sigma}$, we expand $\rho$ close to a given point $\boldsymbol{r}_{0}$,

$$
\rho\left(\delta \boldsymbol{r}+\boldsymbol{r}_{0}\right)=\rho_{0}+\delta \rho,
$$

where $\rho_{0}=\rho\left(\boldsymbol{r}_{0}\right)=\left.\frac{1}{\sqrt{g(\boldsymbol{r})}}\right|_{\boldsymbol{r}=\boldsymbol{r}_{0}}$.

To obtain the variation we carry out a second order perturbation calculation of the heat kernel, using:

$$
\begin{aligned}
e^{-\epsilon\left(\rho_{0}+\delta \rho\right) D}= & e^{-\tilde{\epsilon} D}-\frac{1}{\rho_{0}} \int_{0}^{\tilde{\epsilon}} d s e^{-(\tilde{\epsilon}-s) D} \delta \rho D e^{-s D} \\
& +\frac{1}{\rho_{0}^{2}} \int_{0}^{\tilde{\epsilon}} d s \int_{0}^{s} d s_{1} e^{-(\tilde{\epsilon}-s) D} \delta \rho D e^{-\left(s-s_{1}\right) D} \delta \rho D e^{-s_{1} D}
\end{aligned}
$$

where $\tilde{\epsilon}=\rho_{0} \epsilon$. We assume that the operator $D$ is diagonal in momentum, and that $\delta \rho$ depends on a single coordinate $u$ such as $x$ or $t$ and has an expansion:

$$
\delta \rho(u)=\Sigma_{m=1} c_{m}\left(u-u_{0}\right)^{m}
$$

Explicitly evaluating the heat kernel through second order perturbation series in $\delta \rho$, using the integrals detailed in the Appendix, we find that the leading (in $\epsilon$ ) contributions to $\delta W$ up to two derivatives are given as (1) $\sigma=\sigma(t)$. In this case:

$$
\delta W=\frac{1}{2} \int d t d x \delta \sigma\left(\frac{e^{4 \sigma}}{16 \pi \epsilon}-\frac{1}{24 \pi} \frac{d^{2} \sigma}{d t^{2}}\right)
$$

(2) $\sigma=\sigma(x)$. In this case, the leading order $\epsilon$ contribution reads:

$$
\delta W=\frac{1}{2} \int d t d x \delta \sigma\left(\frac{e^{4 \sigma}}{16 \pi \epsilon}-\frac{e^{2 \sigma}\left(\left(\frac{d \sigma}{d x}\right)^{2}+\frac{d^{2} \sigma}{d x^{2}}\right)}{12 \pi^{3 / 2} \sqrt{\epsilon}}\right)
$$

\section{A. Optimized geometry for equal time correlation functions}

Following [9], we search for a profile $\rho(t)$ to minimize the effective action by solving for $\delta W=0$. Equation (12) implies that the optimal $\sigma(t)$ obeys the Liouville equation:

$$
\frac{e^{4 \sigma}}{\epsilon}-\frac{2}{3} \frac{d^{2} \sigma}{d t^{2}}=0
$$

Much as in [9], The solution is given by the standard substitution of the form $\sigma(t)=-\frac{1}{2} \log \mu t$, where $\mu=\sqrt{\frac{3}{\epsilon}}$ we find the optimal metric is given by

$$
d s^{2}=\frac{1}{\mu^{2} t^{2}} d t^{2}+\frac{1}{\mu t}\left(d x^{2}+d y^{2}\right),
$$

This surprising result suggests that indeed some type of a hierarchical tensor network would still be the optimal discrete spacetime configuration even if the field theory we started with is only anistropically scale invariant. It is interesting to note how the combination $t / \sqrt{\epsilon}$ arises naturally in (14). This is a natural scaling: If we consider our path integral with action (2) as describing, e.g., the ground state of the quantum Lifshitz Hamilotnian, and considering the gap scaling as $\frac{1}{L^{2}}$ for a system with spatial extent $L$, we see that we would have to evolve the system during time $T \sim L^{2}$ in order to resolve the low lying states. Setting $\epsilon \sim \frac{1}{L^{2}}$, we get that the time coordinate has to be scaled as $T \sim \frac{L}{\sqrt{\epsilon}}$.

Noting that our theory is invariant to foliation preserving diffeomorphisms, it is possible to uniformize the geometry by using a coordinate $u=2 \sqrt{t}$ (we take $\epsilon=1$ here), the optimal metric can also be written as

$$
d s^{2}=\frac{4}{3 u^{2}}\left(d u^{2}+\sqrt{3}\left(d x^{2}+d y^{2}\right)\right),
$$

which is the $\mathrm{AdS}_{3}$ metric of a Poincaré patch. Thus, a proper MERA-like description is possible for this nonuniformally rescaled Lifshitz theory. Another possibility, hinted by recent work on exact holographic tensor networks [30], is that a nonunitary MERA-like structure may be chosen that features a scale-invariant tensor network for a non-CFT spin chain model.

\section{B. Optimized geometry for dynamical correlation functions}

We turn to address the optimization in the "lateral" direction. In this case our equation is (13):

$$
\frac{e^{4 \sigma}}{16 \pi \epsilon}-\frac{e^{2 \sigma}\left(\left(\frac{d \sigma}{d x}\right)^{2}+\frac{d^{2} \sigma}{d x^{2}}\right)}{12 \pi^{3 / 2} \sqrt{\epsilon}}=0
$$

To solve this equation, we define: $Y(x) \equiv e^{\sigma(x)}$, and note that (17) can be written as:

$$
Y^{\prime \prime}=C Y^{3} ; \quad C=\frac{3 \pi^{1 / 2}}{4 \sqrt{\epsilon}}
$$


This nonlinear equation is equivalent to the system $Y^{\prime}=Z$; $Z^{\prime}=C Y^{3}$, which allows us to find an integral of motion by solving for $\frac{d Z}{d Y}=\frac{C Y^{3}}{Z}$, from which we obtain the integral of motion:

$$
\frac{1}{2} Y^{\prime 2}=C \frac{Y^{4}}{4}+\text { const. }
$$

We can solve this equation for const $=0$, getting:

$$
Y=\frac{\sqrt{2}}{(\sqrt{C} x+\alpha)}
$$

resulting in the metric, written in terms of $Y$ our metric is

$$
d s^{2}=Y^{4} d t^{2}+Y^{2}\left(d x^{2}+d y^{2}\right)
$$

and the leading behavior of the metric at large $x$ is thus:

$$
d s^{2} \approx 4 \frac{d t^{2}}{C^{2} x^{4}}+2 \frac{d x^{2}+d y^{2}}{C x^{2}} .
$$

We emphasize, that as opposed to the usual notion of holographic Lifshitz geometry for this model, where the boundary is $(2+1)$-dimensional, here we deform one of the original dimensions of the $(2+1)$ spacetime and use it as our holographic direction. We stress that the geometry (22) is also suitable for the computation of equal point correlation functions, as long as all points involved are along a single line. On the other hand, (14) may be useful for computing any multipoint equal correlation functions but not dynamical ones.

\section{FINAL REMARKS}

The equal-time and dynamical two-point correlation functions for the quantum Lifshitz model that we consider in this work have been studied in [18] and more recently in [31] where they have been compared with the holographic two-point function. The authors find that the correlation functions match quite well with the scaling obtained from a holographic calculation with a Lifshitz geometry, thereby strengthening our expectation that a tensor network description of the system will inherit the features of a Lifshitz geometry. We find it quite striking that a semiclassical description of correlation functions is obtained for the system, although there is no manifest small parameter like $\hbar$ or a strong/weak coupling duality to drive us into a semi-classical regime in our original setup. Finally, we remark that although we obtained here an optimal geometry for a specific $z=2(2+1)$-dimensional field theory, it is natural to expect that the procedure described here would still work for more general field theories in higher dimensions with arbitrary values of $z$.

\section{ACKNOWLEDGMENTS}

The work was supported in part by United States NSF Grant No. DMR-1918207.

\section{APPENDIX: PERTURBATIVE HEAT KERNEL CALCULATION}

To obtain our equations we carry out a second order perturbation calculation of the heat kernel, using:

$$
e^{-\epsilon\left(\rho_{0}+\delta \rho\right) D}=e^{-\tilde{\epsilon} D}-\frac{1}{\rho_{0}} \int_{0}^{\tilde{\epsilon}} e^{-(\tilde{\epsilon}-s) D} \delta \rho D e^{-s D}+\frac{1}{\rho_{0}^{2}} \int_{0}^{\tilde{\epsilon}} d s \int_{0}^{s} d s_{1} e^{-(\tilde{\epsilon}-s) D} \delta \rho D e^{-\left(s-s_{1}\right) D} \delta \rho D e^{-s_{1} D}
$$

where $\tilde{\epsilon}=\rho_{0} \epsilon$. For convenience, set $\mathbf{r}_{0}=0$ throughout the calculation, and reinstate its value in the end. We assume that the operator $D$ is diagonal in momentum, and that $\delta \rho$ depends on a single coordinate $u$, and has an expansion:

$$
\delta \rho(u)=\Sigma_{m=1} c_{m} u^{m}
$$

Taking $q$ to be the momentum in the $u$ direction and $K$ to be the momentum vector in all other directions, the zeroth order contribution to the heat kernel reads:

$$
A_{0}=\left\langle 0\left|e^{-\tilde{\epsilon} D}\right| 0\right\rangle=\frac{1}{(2 \pi)^{d+1}} \int d^{d} K d q e^{-\tilde{\epsilon} D(K, q)}
$$

The contribution from the first order term in (A1) is

$$
\begin{aligned}
A_{1} & =-\frac{1}{\rho_{0}}\left\langle 0\left|\int_{0}^{\tilde{\epsilon}} e^{-(\tilde{\epsilon}-s) D} \delta \rho D e^{-s D}\right| 0\right\rangle \\
& =-\frac{1}{\rho_{0}} \frac{2 \pi}{(2 \pi)^{d+2}} \int_{0}^{\tilde{\epsilon}} \int d^{d} K d q\left(\Sigma c_{m}\left(i \frac{d}{d q}\right)\right) m_{e}-(\tilde{\epsilon}-s) D(K, q) e^{-s D(K, q)}
\end{aligned}
$$


which can also be expressed in the form:

$A_{1}=-\frac{1}{\rho_{0}} \frac{1}{(2 \pi)^{d+1}} \int_{0}^{\tilde{\epsilon}} \int d^{d} K d q e^{-\tilde{\epsilon} D(K, q)} D(K, q)\left\{\Sigma_{m=1} i^{m} \sum_{h=1}^{m}(-1)^{h} c_{m} B_{h, m}\left((\tilde{\epsilon}-s) D^{\prime}(K, q),(\tilde{\epsilon}-s) D \prime \prime(K, q), \ldots\right)\right\}$

where $B_{h, m}$ are Bell polynomials. In the case we are interested in, due to the time reversal/space inversion symmetry the first non zero contribution comes from $c_{2}=\left.\frac{1}{2} \partial_{u}^{2} \delta \rho\right|_{u=0}$ :

$$
A_{1} \approx \frac{1}{\rho_{0}} \frac{c_{2}}{(2 \pi)^{d+1}} \int d^{d} K d q e^{-\tilde{\epsilon} D(K, q)}\left(-\frac{1}{2} D \prime \prime(K, q) \tilde{\epsilon}^{2}+\frac{1}{3}\left(D^{\prime}(K, q)\right)^{2} \tilde{\epsilon}^{3}\right) .
$$

The second order contribution is given by:

$$
\begin{aligned}
A_{2} & =\frac{1}{\rho_{0}^{2}}\left\langle 0\left|\int_{0}^{\tilde{\epsilon}} d s \int_{0}^{s} d s_{1} e^{-(\tilde{\epsilon}-s) D} \delta \rho D e^{-\left(s-s_{1}\right) D} \delta \rho D e^{-s_{1} D}\right| 0\right\rangle \\
& =\frac{1}{\rho_{0}^{2}} \frac{\Sigma_{n, m} c_{n} c_{m}}{(2 \pi)^{d+1}} \int_{0}^{\tilde{\epsilon}} d s \int_{0}^{s} d s_{1} \int d^{d} K d q\left(\left(i \frac{d}{d q}\right)^{m} e^{-(\tilde{\epsilon}-s) D(K, q)}\right) D(K, q) e^{-\left(s-s_{1}\right) D(K, q)}\left(\left(-i \frac{d}{d q}\right)^{n} D(K, q) e^{-s_{1} D(K, q)}\right) .
\end{aligned}
$$

When applying these formulas to the situation discussed in the main text, we have considered the two cases: (1) $\rho=\rho(t)$ is only time dependent, where we associate the $q$ momentum with the $t$ coordinate and $D=q^{2}+$ $\left(K_{1}^{2}+K_{2}^{2}\right)^{2}$ and, (2) $\rho=\rho(y)$ is only $y$-dependent, where we associate the $q$ coordinate with $y$ and take $D=K_{1}^{2}+\left(K_{2}^{2}+q^{2}\right)^{2}$.

Carrying out the integrals, and expressing $\rho$ and its local derivatives explicitly in terms of the factor $\sigma$, we get Eqs. (12) and (13).
[1] R. Orús, Ann. Phys. (Amsterdam) 349, 117 (2014).

[2] G. Vidal, Phys. Rev. Lett. 99, 220405 (2007).

[3] G. Vidal, Phys. Rev. Lett. 101, 110501 (2008).

[4] R. N. Pfeifer, G. Evenbly, and G. Vidal, Phys. Rev. A 79, 040301 (2009).

[5] B. Swingle, Phys. Rev. D 86, 065007 (2012).

[6] J. Haegeman, T. J. Osborne, H. Verschelde, and F. Verstraete, Phys. Rev. Lett. 110, 100402 (2013).

[7] M. Nozaki, S. Ryu, and T. Takayanagi, J. High Energy Phys. 10 (2012) 193.

[8] M. Miyaji, T. Numasawa, N. Shiba, T. Takayanagi, and K. Watanabe, Phys. Rev. Lett. 115, 171602 (2015).

[9] P. Caputa, N. Kundu, M. Miyaji, T. Takayanagi, and K. Watanabe, Phys. Rev. Lett. 119, 071602 (2017).

[10] P. Caputa, N. Kundu, M. Miyaji, T. Takayanagi, and K. Watanabe, J. High Energy Phys. 11 (2017) 097.

[11] M. Miyaji, T. Takayanagi, and K. Watanabe, Phys. Rev. D 95, 066004 (2017).

[12] Y. Sato and K. Watanabe, J. High Energy Phys. 11 (2019) 132.

[13] G. Jafari, A. Naseh, and H. Zolfi, Phys. Rev. D 101, 026007 (2020).

[14] M. Ghodrati, J. High Energy Phys. 02 (2020) 052.

[15] P. Caputa and I. MacCormack, J. High Energy Phys. 01 (2021) 027.
[16] G. Evenbly and G. Vidal, Phys. Rev. Lett. 115, 180405 (2015).

[17] G. Evenbly and G. Vidal, J. Stat. Phys. 145, 891 (2011).

[18] E. Ardonne, P. Fendley, and E. Fradkin, Ann. Phys. (Amsterdam) 310, 493 (2004).

[19] E. Fradkin and J. E. Moore, Phys. Rev. Lett. 97, 050404 (2006).

[20] S. Isakov, P. Fendley, A. Ludwig, S. Trebst, and M. Troyer, Phys. Rev. B 83, 125114 (2011).

[21] In terms of the ADM metric commonly used in the literature, $g_{t t}=\frac{1}{N^{2}}$.

[22] T. Griffin, P. Hořava, and C. M. Melby-Thompson, J. High Energy Phys. 05 (2012) 010.

[23] M. Baggio, J. de Boer, and K. Holsheimer, J. High Energy Phys. 07 (2012) 099.

[24] M. Baggio, J. de Boer, and K. Holsheimer, J. High Energy Phys. 01 (2012) 058.

[25] I. Arav, S. Chapman, and Y. Oz, J. High Energy Phys. 02 (2015) 078.

[26] I. Arav, Y. Oz, and A. Raviv-Moshe, J. High Energy Phys. 03 (2017) 088.

[27] A. O. Barvinsky, D. Blas, M. Herrero-Valea, D. V. Nesterov, G. Pérez-Nadal, and C. F. Steinwachs, J. High Energy Phys. 06 (2017) 063. 
[28] D. Nesterov and S. N. Solodukhin, Nucl. Phys. B842, 141 (2011).

[29] P. Francesco, P. Mathieu, and D. Sénéchal, Conformal Field Theory (Springer Science \& Business Media, New York, 2012).
[30] R. N. Alexander, G. Evenbly, and I. Klich, arXiv:1806 .09626.

[31] V. Keränen, W. Sybesma, P. Szepietowski, and L. Thorlacius, J. High Energy Phys. 05 (2017) 033. 